\title{
Eficacia de la Hipnosis en Psicología del Deporte: una revisión sistemática.
}

\section{Efficacy of Hypnosis in Sport Psychology: a systematic review.}

\section{Eficácia da Hipnose na Psicologia do Desporto: Uma Revisão Sistemática.}

\author{
Franquelo, M. A. ${ }^{1}$, Hernández-Mendo, A. ${ }^{1}$ Capafons, A. ${ }^{2}$. \\ ${ }^{1}$ Universidad de Málaga, Málaga (España); ${ }^{2}$ Universitat de Valencia, Valencia (España).
}

\begin{abstract}
RESUMEN
La hipnosis es un conjunto variado de procedimientos que posee abundante evidencia científica sobre su utilidad como técnica coadyuvante en el tratamiento de distintos trastornos, de índole médica o psicológica especialmente en adultos. No obstante, en el ámbito de la Psicología del Deporte la evidencia disponible parece escasa, y no siempre basada en estudios rigurosos desde un punto de vista metodológico. Por este motivo, este estudio presenta una revisión sistemática según la Declaración Prisma sobre la eficacia de la hipnosis en el deporte y el ejercicio/práctica deportiva. Los objetivos son describir los resultados de este conjunto de técnicas sobre el rendimiento en deportistas y practicantes de ejercicio físico, y mostrar la potencia estadística del conjunto de estudios y su rigor metodológico. Se incluyeron un total de 45 estudios, que revelaron, en su mayoría, los efectos positivos del uso de la hipnosis frente a otras técnicas utilizadas. Con respecto a los criterios metodológicos, la mayoría de los estudios cumplieron con los criterios establecidos, a excepción de evaluación de la sugestionabilidad hipnótica y medida del tamaño del efecto. Estos resultados ponen de relevancia la importancia de continuar contribuyendo al desarrollo de la hipnosis en Psicología del Deporte, visto que los posibles efectos positivos y las ventajas que puede presentar la misma presentan aún poca evidencia científica.
\end{abstract}

Palabras clave: Hipnosis, deporte, eficacia, rendimiento.

\section{ABSTRACT}

Hypnosis is a technique that has a multitude of scientific evidence in favor of its efficacy as an adjuvant technique in the treatment of different disorders - medical or psychological nature- in children and adults, and more specifically, also in the field of sport psychology, although this evidence has not always been accompanied by rigorous methodological procedures. For this reason, this study presents a systematic review on the efficacy of hypnosis in sport and exercise, describing the results of this technique on performance in athletes and physical activity practitioners, and exploring the statistical power of the set of studies. A total of 45 studies were included, most of which revealed the positive effects of the use of hypnosis compared to other techniques used. With respect to the methodological criteria, most of the studies met the established criteria, with the exception of the assessment of hypnotic suggestibility and the measurement of effect size, both of which are of utmost importance. These results highlight the importance of continuing to contribute to the development of hypnosis in the discipline, given the positive effects and advantages that hypnosis can have.

Keywords: Hypnosis, sport, effectiveness, performance.

\section{RESUMO}

A hipnose é um conjunto variado de procedimentos que tem provas científicas abundantes sobre a sua utilidade como técnica adjuvante no tratamento de várias doenças, especialmente em adultos. No entanto, no campo da Psicologia 


\section{Franquelo et al.}

do Desporto as provas disponíveis parecem ser escassas, e nem sempre baseadas em estudos rigorosos de um ponto de vista metodológico. Por esta razão, este estudo apresenta uma revisão sistemática de acordo com a Declaração Prisma sobre a eficácia da hipnose no desporto e na prática de exercício/esporte. Os objectivos são descrever os resultados deste conjunto de técnicas sobre o desempenho em atletas e praticantes de actividade física, e mostrar o poder estatístico do conjunto de estudos e o seu rigor metodológico. Foram incluídos um total de 45 estudos. A maioria deles revelou os efeitos positivos do uso da hipnose em comparação com outras técnicas. No que diz respeito aos critérios metodológicos, a maioria dos estudos cumpriu os critérios estabelecidos, com excepção da avaliação da sugestibilidade hipnótica e da medição da dimensão do efeito. Estes resultados sublinham a importância de continuar a contribuir para o desenvolvimento da hipnose na Psicologia do Desporto, dado que os possíveis efeitos positivos e vantagens da hipnose ainda apresentam poucas provas científicas.

Palavras chave Hipnose, Desporto, eficacia, desempenho.

\section{INTRODUCCIÓN}

Existen multitud de definiciones relativas a la naturaleza de la hipnosis que aluden a distintos aspectos y connotaciones. Atendiendo a Hilgard (1977), se considera un estado disociado que implica una desconexión entre los procesos conscientes e inconscientes, lo que parece facilitar la receptividad y accesibilidad de la mente inconsciente a la sugestión. Spiegel y Spiegel (1987), por otra parte, la conciben como un estado psicofisiológico en el que la conciencia periférica se ve reducida y la concentración se focaliza. Kilhstrom (1998) alude al aspecto psicosocial, defendiendo que se trata de una interacción social donde una persona (hipnotizado) responde a las sugestiones dadas por otra persona (hipnotizador).

Por este motivo, y ante la dificultad de ponerse de acuerdo, la División 30 de la APA propuso en 2004 una definición ampliamente aceptada y consensuada por las principales organizaciones, que conceptualiza la hipnosis como un procedimiento donde un profesional/investigador del área de salud suscita una serie de cambios en las percepciones, sensaciones, pensamientos o conductas de un sujeto (APA, 2004). Posteriormente, en 2014, el Comité Ejecutivo de la División 30 de la APA propone una nueva definición que afirma que "la hipnosis es un estado de conciencia que implica la atención focalizada y conciencia periférica reducida, caracterizado por una mayor capacidad de respuesta a la sugestión" (APA, 2014). Por otra parte, la Asociación para el Avance de la Hipnosis Experimental y Aplicada (AAHEA) (AAHEA, 2017) aunó las ideas de distintos autores para definir la hipnosis como constructo social con distintas connotaciones que han variado a través del tiempo. De este modo, y según la AAHEA (2017), es común utilizar el término para referir una interacción social en la que las personas que participan (hipnotizadoras e hipnotizadas) asumen roles basados en formas de influencia social. De esto se deduce que los participantes asumen un acuerdo, que puede ser explícito o implícito, que dará lugar a un proceso donde la persona hipnotizadora tratará de influir, mediante sugestiones hipnóticas, en los sentimientos, pensamientos y conductas de la persona hipnotizada, que activará el rol asociado a su concepción de hipnosis. Además de esto, la hipnosis también constituye un campo de investigación básica $\mathrm{y}$ aplicada (AAHEA, 2017).

Con respecto a la eficacia de la hipnosis, existe multitud de investigación acorde con la mejora de la eficacia y la eficiencia de los tratamientos a los que se adjunta como técnica coadyuvante, para el tratamiento de multitud de trastornos, ya sean de índole médica o psicológica, en niños y adultos, tal y como se evidencia en distintas revisiones (p. e. Molina del Peral et al., 2016a; Molina del Peral et al., 2016b). De forma más concreta, siguiendo los criterios de Chambless y Hollon (1998), ha demostrado ser eficaz en el manejo del dolor con un efecto moderado - alto (Montgomery et al., 2000); en el tratamiento de la ansiedad, cuando se combina con el tratamiento cognitivo - conductual (Schoenberger et al., 1997); probablemente eficaz en el tratamiento de la obesidad y el tabaquismo (p. e. Elkins y Rajab, 2004; Elkins et al., 2006; Green, 1996; Lynn et al., 1993; Mendoza, 2000); en el tratamiento de trastornos gastrointestinales cuando se combina con otras técnicas (p. e. Gonsalkorale et al., 2002; Gonsalkorale y Whorwell, 2005; Palsson, 2006; Simrén, 2006; Whitehead, 2006; Whorwell, 2006); en la preparación a la cirugía (p. e. Blankfield, 1991; Faymonville et al., 1997; Faymonville et al., 1999; Montgomery et al., 2002; Pinnel y Covino, 2000); y en otras muchas áreas (p. e. manejo de ansiedad, depresión, estrés post-traumático, etc.).

Sin embargo, es importante que el bagaje científico que avala su eficacia y eficiencia siga creciendo, especialmente en las áreas donde ya resulta prometedora. Esto es así, principalmente porque, a pesar de que existe gran cantidad de investigación, en 


\section{Hipnosis en Psicología del Deporte}

algunos de los casos no se cumplen criterios metodológicos estrictos (Hernández-Mendo et al., 2017). Por este motivo, sigue siendo necesario el desarrollo de estudios e investigaciones con una perspectiva más precisa en algunos campos, como en el área de la Psicología del Deporte, donde es aún necesaria una mayor exploración (Barker et al., 2013). En este ámbito, a pesar de obtener resultados optimistas, existen diversos estudios con evidentes lagunas metodológicas (Barker et al., 2013), ya que una parte de la investigación está referida a estudios con muestras muy reducidas, estudios de caso o relatos de experiencias propias (Hernández-Mendo et al., 2017). A pesar de ello, estas situaciones no indican que la hipnosis en esta área constituya un tratamiento ineficaz (algunos cumplen con criterios metodológicos estrictos, véanse los propuestos por Chambless y Hollon en 1998, en relación con estudios de caso $\mathrm{n}=$ 1), sino que, más bien, indican que se debe continuar investigando y profundizando en este ámbito (Lynn et al., 2000), tal y como se señalaba anteriormente.

Sin embargo, pese a que el uso de la hipnosis en Psicología del Deporte no cuenta con tanto aval como puede hacerlo el uso de la hipnosis en Psicología (Hernández-Mendo et al., 2017), se pueden encontrar algunos ejemplos de utilización de la hipnosis para la mejora del rendimiento, ya sea directamente (p. e. Winter (1986) y Schreiber (1991) en baloncesto, o Lindsay et al., (2005) y Zurita et al. (2014) en ciclismo, entre otros) o indirectamente (p. e. Cracium y Szatmari (2007) o Hernández-Mendo et al., (2017), entre otros).

Llegados a este punto, dada la utilidad puesta de relieve en diversos estudios, y ante la necesidad de valorar la situación actual de la hipnosis en este campo de aplicación, los objetivos del estudio son: (1) siguiendo los principios de la declaración Prisma, seleccionar aquellos estudios que se centren en explorar la eficacia de esta técnica sobre el rendimiento en deportistas de distintas modalidades y practicantes de ejercicio físico, bien mediante un efecto directo sobre el rendimiento, o mediante un efecto mediado a través de otras variables psicosociales, y describir los resultados de los mismos; y (2) explorar la potencia estadística del conjunto de estudios, así como sus limitaciones.

\section{MATERIAL Y MÉTODOS}

Se realizó una revisión sistemática, siguiendo las indicaciones efectuadas por PRISMA (Prefered Reporting Items for Systematic Review and MetaAnalyses), donde se analizaron artículos originales y de revisión publicados entre los años 1925 y 2020. Las bases de datos utilizadas fueron: Web of Science, Scopus, PSICODOC, PsycARTICLES, PSYCINFO y Pubmed. La búsqueda se realizó en inglés y español, utilizando los descriptores ("hypnosis"/"hipnosis") AND ("ejercicio"/"exercise" OR "deporte"/"sport") AND ("eficacia"/"efficacy" "rendimiento"/"performance"). Los resultados obtenidos se limitaron por idioma (español e inglés) y por tipo de documento (se eliminaron meetings, patentes y biografías). Además, también se incluyeron estudios procedentes de otras fuentes (p. e. referencias de artículos utilizados).

\section{Criterios de inclusión y de exclusión}

Este estudio tuvo como objetivo describir los resultados de la hipnosis sobre el rendimiento en deportistas y practicantes de ejercicio físico, o sobre variables o respuestas que medien o afecten al rendimiento deportivo y/o la práctica físico deportiva. Los criterios de inclusión fueron:

Criterio 1: estudios de caso, revisiones, estudios experimentales o cuasiexperimentales.

Criterio 2: en español o inglés.

Criterio 3: que la hipnosis forme parte de la intervención, orientada a mejorar el rendimiento (pudiendo ser la relación entre hipnosis y rendimiento pudo ser directa, p. e. examinar los efectos de un programa de hipnosis sobre el rendimiento $o$ mediada/indirecta, p. e. examinar los efectos de un programa de hipnosis en la reducción de la ansiedad o la mejora del autoconcepto).

Los criterios de exclusión fueron:

Criterio 1: meetings, patentes y biografías.

Criterio 2: que el estudio no incluya variables o respuestas que medien o afecten al rendimiento deportivo y/o la práctica físico - deportiva.

Tal y como se refleja en la Figura 1, se obtuvieron un total de 583 resultados procedentes de las bases de datos consultadas. Además, se identificaron un total de 43 resultados procedentes de otros recursos y fuentes (p. e. referencias de estudios relevantes). Esto produjo un total de 626 resultados, de los que, al eliminar duplicados, quedan 380. Así mismo, se eliminaron un total de 39,23 por estar en un idioma distinto al español o inglés, y 16 por pertenecer a un tipo de documento que quedaba fuera del estudio. Esto generó un total de 587 resultados que fueron examinados en mayor detalle. Tras consultar los resúmenes y abstracts, se eliminaron un total de 296 por no enmarcarse en el objeto de estudio o cumplir los criterios de inclusión (p. e. utilizar la hipnosis como técnica con fines clínicos o terapéuticos ajenos al ámbito deportivo en términos de rendimiento; no utilizar la hipnosis como técnica, utilizando otras técnicas en su lugar). Finalmente, se incluyeron un total de 45 estudios. 
Franquelo et al.

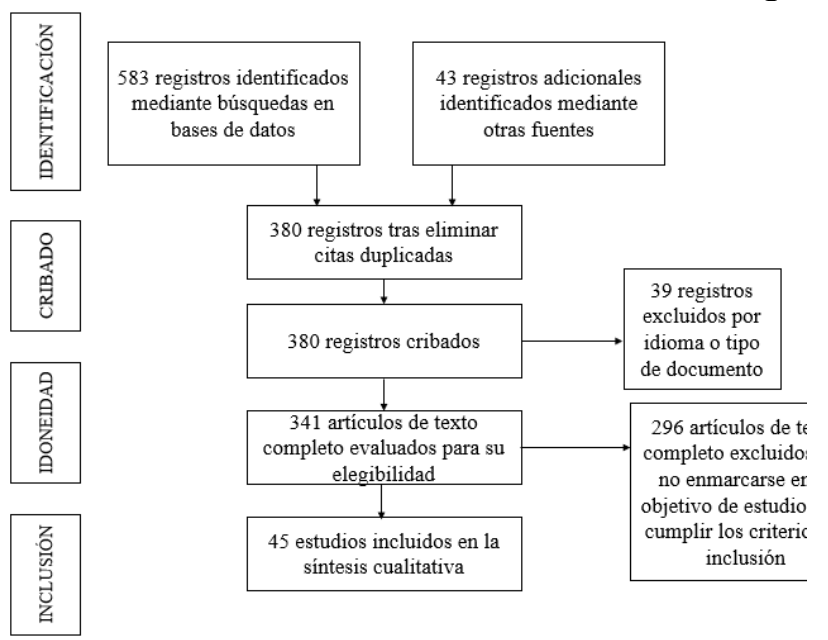

Figura 1. Flujo de información PRISMA.

\section{RESULTADOS}

Los resultados obtenidos (descritos en la Tabla 1) se pueden agrupar atendiendo a distintos tipos de variables. De esta forma, en tenis, Anchique (2006) pretendió evaluar, la utilidad de la autosugestión positiva en el incremento de la efectividad de primer y segundo servicio. No encontró diferencias significativas en ambas mediciones entre el primer y el segundo servicio, pero sí reportó diferencias significativas a favor de la efectividad total, encontrando además una disminución en las dobles faltas. En esta línea, Greer y Engs (1986) concluyeron que instrucciones de práctica mental junto a relajación progresiva e hipnosis no resultaron ser más eficaces que la práctica tradicional (explicación y demostración) en un grupo de principiantes en clases de tenis.

En cricket, Barker y Jones (2006) exploraron el incremento de la autoeficacia con un jugador semi profesional de cricket mediante el uso de hipnosis, perfeccionamiento y automodelado, encontrando diferencias significativas en los niveles de autoeficacia pre y post intervención, además de un incremento del rendimiento.

Se encuentran distintos estudios en cuanto a fútbol. Así Newmark y Bogacki (2005) plantearon, por una parte, una intervención con hipnosis para un futbolista que afirmó devolver demasiado pronto el balón, logrando mejorar su rendimiento (y contribuyendo en el tratamiento de su depresión); por otra parte, plantearon una intervención para el control de la ira con un joven futbolista, logrando controlar sus ataques y dirigiendo su ira hacia el balón. Del mismo modo, Barker y Jones (2008) analizaron la eficacia de la hipnosis como intervención para reducir el afecto negativo y aumentar la autoeficacia, la confianza, el afecto positivo y el rendimiento en un jugador de fútbol profesional, encontrando resultados que indicaron que la hipnosis puede ser utilizada para la mejora de la autoeficacia y el rendimiento deportivo. Así Barker et al., (2010) estudiaron los efectos de la hipnosis sobre la autoeficacia y el rendimiento en fútbol, demostrando que la hipnosis puede ser utilizada para mejorar y mantener la autoeficacia y el rendimiento. También Hernández-Mendo, et al. (2017) reportaron un estudio con un jugador de fútbol profesional con el que se realizó una intervención hipnótica (solicitada por falta de eficacia en la consecución de goles) que concluyó con un claro aumento en el número de goles marcados en la temporada (siendo la temporada en la que más goles marca).

En ciclismo, Lindsay et al., (2005) evaluaron la eficacia de una intervención hipnótica sobre los estados de flow y sobre el rendimiento en ciclistas, encontrando resultados que indicaron una mejora del rendimiento, así como un incremento en los sentimientos y pensamientos relacionados con el flow. Fernández et al. (2012) evaluaron la eficacia de la hipnosis en la resistencia a esfuerzos máximos, encontrando que un incremento en las sesiones de intervención con hipnosis se correspondía con un incremento en la resistencia a situaciones de máximo esfuerzo. En esta línea, Fernández et al., (2013) estudiaron la eficacia de la hipnosis para modificar variables psicológicas y fisiológicas en ciclistas durante la realización de pruebas de esfuerzo, encontrando una tendencia positiva en las puntuaciones de las variables psicológicas en el grupo que utilizó hipnosis. Por último, Zurita et al. (2014) estudiaron la eficacia de la aplicación de un programa de hipnosis en ciclismo, encontrando una mejora duradera de la fuerza, manteniéndose constante la percepción subjetiva de fatiga.

En relación con ejercicios de fuerza o la resistencia, el trabajo de Roush (1951) examinó si el rendimiento físico puede verse modificado a través de tres estados: hipnótico, post-hipnótico y vigilia; encontrando diferencias significativas en fuerza evaluada con dinamómetro manual y de brazo, y también en test de resistencia. Orne (1959), por otra parte, probó que los participantes podrían rendir mejor con un conjunto de instrucciones motivacionales bien dirigidas que bajo hipnosis en una prueba de resistencia al peso. London y Fuhrer (1961) encontraron que el rendimiento (dinamómetro, resistencia al peso y prueba de temblor) en estudiantes con baja sugestionabilidad era mayor que el de sujetos altamente sugestionables, llegando a concluir que la hipnosis, por sí misma, no tiene efectos sobre el rendimiento. En esta línea, Rosenhan y London (1963) examinaron, utilizando distintas tareas de fuerza y resistencia, si se producían diferencias en el rendimiento cuando los participantes descubrían que se iba a utilizar hipnosis en el experimento; encontraron que no se producían diferencias con un dinamómetro manual y en una tarea de resistencia, pero sí se encontraron diferencias con una prueba de 


\section{Hipnosis en Psicología del Deporte}

temblor. Levitt y Brady (1964) desarrollaron un estudio con tres condiciones experimentales (hipnosis, hipnosis + analgesia hipnótica, vigilia + instrucciones motivacionales + recompensa económica) para explorar los efectos sobre la resistencia muscular, encontrando que no se producen efectos significativos entre las condiciones. Morgan et al., (1973) encontraron que sugestiones hipnóticas con respecto a trabajo duro, ligero o moderado evocaban percepciones y cambios fisiológicos (percepción de esfuerzo, ritmo cardíaco y estabilidad). Albert y Williams (1975) pretendieron examinar los efectos de sugestiones post-hipnóticas sobre resistencia, esfuerzo percibido (escala Borg) y frecuencia cardíaca, encontrando que las sugestiones de fatiga se correspondían con un tiempo de resistencia acortado, pero las sugestiones facilitadoras no tenían efectos. En esta línea, Jackson et al., (1979) diseñaron un estudio con cinco condiciones: control, solo hipnosis, solo instrucciones motivacionales, baja susceptibilidad a la hipnosis + instrucciones motivacionales y alta susceptibilidad a la hipnosis + instrucciones motivacionales, a fin de estudiar sus efectos sobre la resistencia, encontrando incrementos significativos en la resistencia de los grupos con solo instrucciones motivacionales y alta susceptibilidad a la hipnosis + instrucciones motivacionales; así mismo, encontraron un incremento en la ventilación máxima del grupo con alta susceptibilidad a la hipnosis (en comparación con el grupo control) y un incremento significativo en las concentraciones de lactato (en comparación con el grupo con solo motivación y con baja susceptibilidad a la hipnosis). Howard y Reardon (1986) desarrollaron un estudio con cuatro condiciones experimentales (enfoque cognitivo - hipnótico en imaginería (CHI), reestructuración cognitiva, hipnosis y control) para examinar los efectos de estos tratamientos sobre el rendimiento neuromuscular, el crecimiento muscular, la reducción de ansiedad y la mejora del autoconcepto en levantadores de pesos, encontrando que los sujetos del grupo CHI mostraron una mejora sustancial en todas las variables $\mathrm{y}$, además, que esta mejora perduraba en el tiempo.

Atendiendo a variables de tipo cognitivo, Young (1925) utilizó distintas pruebas (movimiento del brazo -de izquierda a derecha- sobre un sector, dinamómetro, prueba de estabilidad, presión sobre la piel, deletrear al revés, abecedario al revés, amplitud de dígitos, suma acumulativa, memoria para sílabas $\sin$ sentido, memoria en asociaciones adjetivosustantivo, amplitud de memoria, memoria para asociaciones misceláneas, asociaciones lógicas, libre asociación y discriminación de manchas), encontrando resultados dispersos entre sujetos en vigilia e hipnotizados y concluyendo, finalmente, que no se producían cambios en la habilidad a través de estos dos estados. Fehr y Stern (1967) encontraron que sujetos hipnotizados presentaban un comportamiento menos receptivo ante tareas de vigilancia, presentando un tiempo de reacción más lento y una mayor latencia de respuesta. London et al., (1968) encontraron pocas diferencias en el rendimiento de sujetos sugestionables y poco sugestionables, mejorando ambos después de ser hipnotizados o exhortados; así mismo, el rendimiento del grupo sugestionable se vio más afectado por la hipnosis y la exhortación que el rendimiento de los poco sugestionables. Rader (1972) encontró distintos tiempos de reacción en función de la técnica utilizada con los participantes (hipnosis, hipnosis con instrucciones motivacionales, instrucciones motivacionales o control), encontrando que la hipnosis por sí sola no presentaba especial habilidad para mejorar los tiempos de reacción en comparación con la motivación.

Con respecto al aprendizaje, Edmonston y Marks (1967) no encontraron efectos significativos de la hipnosis ni de instrucciones motivacionales sobre el aprendizaje kinestésico, llegando incluso a encontrar que podía producir interferencias en el aprendizaje de una tarea. En esta línea, Arnold (1971) tampoco encontró resultados significativos sobre el aprendizaje motor de sugestiones positivas (con o sin hipnosis), ni tampoco diferencias en base a la hipnotizabilidad de los sujetos. En contraposición, Jalene y Wulf (2014) encontraron que la hipnosis podía tener un impacto positivo en el aprendizaje motor, dejando la puerta abierta al estudio de los mecanismos por los que se produce este efecto.

Por otra parte, en cuanto a baloncesto, Winter (1986) reportó el caso de una jugadora de baloncesto que, tras una intervención combinada de hipnosis con otras técnicas, consiguió aumentar en un 23\% su eficacia de tiro en lanzamientos libres. Así mismo, Schreiber (1991) encontró que la hipnosis podía mejorar el rendimiento de tiro en jugadores y jugadoras de baloncesto, encontrando que el grupo que trabaja con hipnosis acumulaba un mayor número de puntos con respecto al grupo control. Pates et al., (2001a) investigaron los efectos de la hipnosis sobre el set and jump, encontrando que esta podía mejorar el rendimiento, así como las sensaciones asociadas al rendimiento máximo de los jugadores, durante el tiempo que duró la intervención. Pates et al., (2002) llevaron a cabo una investigación con jugadores de baloncesto, en la que, utilizando técnicas hipnóticas, consiguieron mejorar el rendimiento en triples de los participantes, así como los sentimientos y cogniciones asociados a los estados de flow. Por último, Newmark y Bogacki (2005) plantearon una intervención con hipnosis para un jugador de baloncesto que reportó disparos inexactos y una defensa ineficaz, logrando mejorar su precisión de tiro y su defensa.

En el caso del golf, Nelson (1980) no encontró resultados significativos con respecto a beneficios de la hipnosis (tampoco relajación ni ensayo mental) en 


\section{Franquelo et al.}

golfistas. Sin embargo, Pates y Maynard (2000) examinaron los efectos de una intervención hipnótica en golf, encontrando una mejora del rendimiento en el chip de golf, y también ayudó a los participantes a mantenerse confiados, relajados y con la situación bajo control. Además, en algunos de los participantes, la intervención contribuyó a que experimentasen una mayor sensación de flow. En esta línea, Pates et al., (2001b) investigaron sobre los efectos de la hipnosis en golf, encontrando que el uso de esta técnica incrementa el rendimiento de los participantes, así como percepción sobre el estado de flow, relatando además que se percibían más tranquilos, seguros y concentrados. Newmark y Bogacki (2005) plantearon una intervención con un golfista amateur con la que consiguieron mejorar su golpeo, aumentando el rendimiento. Pates $(2013,2014)$, a su vez, examinaron los efectos de una intervención con hipnosis jugadores de golf profesional, encontrando que dicha intervención disminuyo el promedio de golpes y aumento el promedio de puntos, relatando, además, un mayor control de emociones, pensamientos y percepciones. Pates (2019), en contraposición, encontró que la intervención propuesta hace disminuir el promedio. Por último, Pates y Cowen (2013) encontraron nuevamente una mejora consistente en el rendimiento de un golfista, además de un incremento en la intensidad de las experiencias de flow durante la competición.

En cuanto a bádminton, Pates y Palmi (2002) examinaron los efectos de la hipnosis sobre el rendimiento en el servicio corto de bádminton y sobre los estados de flow, encontrando que el rendimiento en jugadoras competitivas aumentaba, así como sus percepciones en estados de flow, y sus sensaciones de tranquilidad, relajación, determinación, felicidad y concentración.

Atendiendo al tiro con arco, por su parte, Robazza y Bortoli (1995) combinaron técnicas de hipnosis activo - alerta e hipnosis tradicional en sesiones de entrenamiento mental para incrementar el rendimiento en el tiro con arco, encontrando que este entrenamiento resulta eficaz. En cuanto a tiro deportivo (rifles de aire comprimido), Mattle et al., (2020) encontraron que una sola intervención con hipnosis producía pequeños efectos positivos o mejoras en el rendimiento de la mayoría de los participantes.

En el caso de la escalada, Morton (2003) relató su propia experiencia con auto-hipnosis para enfrentarse a los distintos desafíos que se le plantean durante distintas expediciones de escalada, concluyendo que la técnica le ayudó a manejar y reducir la ansiedad, así como a aproximarse a la zona de funcionamiento óptimo, incrementando la autoconfianza; además de una estrategia efectiva para dirigir y organizar la energía, y eliminar pesadillas y miedos recurrentes.

Por otro lado, en piragüismo, Fernández et al. (2004) evaluaron la eficacia de la hipnosis (junto a la terapia de aceptación y compromiso - ACT-) para la mejora de la fuerza física en piragüistas, hallando una mejora significativa en el número de repeticiones de remo inclinado.

Con respecto al judo, Cracium y Szatmari (2007) examinaron los efectos de la hipnosis activo-alerta sobre los estados de ánimo de practicantes de judo, reportando un cambio destacado en los estados emocionales, con una disminución de la tensiónansiedad, la depresión, el enfado, el cansancio y la confusión, así como un aumento en el vigor-actividad, presentando un perfil tipo iceberg tras la intervención, característico de deportistas de éxito.

En corredores (de distintas disciplinas), Nelson (1980) no encontró resultados significativos con respecto a beneficios de la hipnosis (tampoco relajación ni ensayo mental) en corredores. Por otra parte, Hernández-Mendo et al. (2017) plantearon una intervención enfocada a reducir el dolor en un corredor de atletismo tras un accidente automovilístico, logrando una reducción en el dolor y una recuperación más temprana. Del mismo modo, Hernández-Mendo et al., (2017) diseñaron dos programas de intervención (distintas demandas), ambos exitosos y separados por un intervalo temporal de 10 años, para una corredora de montaña y ultra-trail, enfocados al trabajo, en primera instancia, de un trastorno de la conducta alimentaria (consiguiendo eliminar los indicios de ansiedad y las conductas de provocación de vómito) y, en segundo lugar, debido a la imposibilidad de beber agua durante la carrera (logrando que la atleta consiguiera beber agua).

Por último, en salto con pértiga, Winter (1986), reportó el caso de un saltador de pértiga que, tras una intervención de siete sesiones con hipnosis combinada con otras técnicas, consiguió su mejor marca personal. Y Hernández-Mendo et al. (2017) reportó un diseño de caso único con una saltadora de pértiga, donde tras solicitar una intervención tras lesión, se realizó una intervención hipnótica que concluyó con una mejora del rendimiento (aumento en la altura del salto). 


\section{Hipnosis en Psicología del Deporte}

Tabla 1. Características de los estudios incluidos en la revisión.

\begin{tabular}{|c|c|c|c|}
\hline Estudio & Objetivo & Muestra y diseño & Resumen de los principales hallazgos \\
\hline $\begin{array}{l}\text { Young } \\
(1925)\end{array}$ & $\begin{array}{l}\text { Examinar las diferencias en } \\
\text { rendimiento a través de distintas } \\
\text { pruebas, en condiciones lo más } \\
\text { similares posibles, entre sujetos en } \\
\text { vigilia y en hipnosis. }\end{array}$ & $\begin{array}{l}22 \text { estudiantes } \\
\text { universitarios: } 16 \text { en el } \\
\text { grupo de hipnosis y } 6 \text { en } \\
\text { grupo control. }\end{array}$ & $\begin{array}{l}\text { Se encontraron resultados dispares a } \\
\text { través de las distintas tareas. }\end{array}$ \\
\hline $\begin{array}{l}\text { Roush } \\
(1951)\end{array}$ & $\begin{array}{l}\text { Examinar si el rendimiento físico } \\
\text { puede ser incrementado durante el } \\
\text { "estado }{ }^{1} \text { " hipnótico (EH) o post- } \\
\text { hipnótico (EP) en comparación con } \\
\text { el estado de vigilia (EV). }\end{array}$ & $\begin{array}{c}11 \text { mujeres para la primera } \\
\text { serie; } 5 \text { hombres y } 10 \\
\text { mujeres para la segunda } \\
\text { serie. }\end{array}$ & $\begin{array}{l}\text { En la primera serie de test, no se } \\
\text { encontraron diferencias significativas } \\
\text { entre el EH y el EV con respecto al } \\
\text { dinamómetro manual y el test de } \\
\text { resistencia, pero se encontró un } \\
\text { incremento en el dinamómetro de brazo } \\
\text { para todos los sujetos en el EH. En la } \\
\text { segunda serie se encontraron diferencias } \\
\text { significativas con el dinamómetro de } \\
\text { brazo entre el EH y el EV y muy } \\
\text { significativas entre el EP y el EV. No se } \\
\text { encontraron diferencias entre el EH y el } \\
\text { PH. Con respecto al dinamómetro } \\
\text { manual, se encontraron diferencias muy } \\
\text { significativas entre el EH y el EV; } \\
\text { significativas entre el EP y el EV; y no se } \\
\text { encontraron entre el EH y el EP. Con } \\
\text { respecto al test de resistencia, se } \\
\text { encontraron diferencias significativas } \\
\text { entre el EH y el EV; no se encontraron } \\
\text { entre el EH y el EP. }\end{array}$ \\
\hline Orne (1959) & $\begin{array}{l}\text { Examinar si existe aumento de } \\
\text { capacidad física en hipnosis y } \\
\text { probar la hipótesis de que pueda ser } \\
\text { considerado por un aumento en la } \\
\text { motivación. }\end{array}$ & 11 estudiantes. & $\begin{array}{l}\text { Los resultados mostraron que los } \\
\text { participantes podrían rendir mejor sin } \\
\text { hipnosis que si se les dieran las } \\
\text { instrucciones motivacionales adecuadas. }\end{array}$ \\
\hline $\begin{array}{l}\text { London y } \\
\text { Fuhrer } \\
(1961)\end{array}$ & $\begin{array}{c}\text { Explorar las interacciones entre } \\
\text { hipnosis, motivación y las } \\
\text { demandas experimentales } \\
\text { inherentes al contexto experimental } \\
\text { en un solo experimento } \\
\text { (dinamómetro, resistencia al peso y } \\
\text { prueba de temblor). }\end{array}$ & $\begin{array}{c}32 \text { estudiantes } \\
\text { universitarias divididas en } \\
\text { dos grupos: alta y baja } \\
\text { sugestionabilidad. }\end{array}$ & $\begin{array}{c}\text { Los resultados indicaron que el } \\
\text { rendimiento del grupo baja } \\
\text { sugestionabilidad fue superior al } \\
\text { altamente sugestionable. Ambos grupos } \\
\text { se vieron altamente afectados por las } \\
\text { indicaciones, siendo su rendimiento } \\
\text { mucho mejor cuando se les daban. } \\
\text { Concluyeron que el uso de la hipnosis, } \\
\text { por sí solo, no tiene efectos significativos } \\
\text { sobre el rendimiento. }\end{array}$ \\
\hline $\begin{array}{l}\text { Rosenhan y } \\
\text { London } \\
(1963)\end{array}$ & $\begin{array}{l}\text { Investigar los efectos relativos del } \\
\text { conocimiento de que un } \\
\text { experimento implica hipnosis sobre } \\
\text { el rendimiento, utilizando } \\
\text { dinamómetro manual, tarea de } \\
\text { resistencia y de temblor. }\end{array}$ & $\begin{array}{l}32 \text { estudiantes clasificados } \\
\text { en dos grupos según su } \\
\text { sugestionabilidad. }\end{array}$ & $\begin{array}{l}\text { Las diferencias entre los grupos de alta y } \\
\text { baja sugestionabilidad eran más } \\
\text { marcadas antes de saber que el } \\
\text { experimento implicaba hipnosis, siendo } \\
\text { menos evidentes después. En el caso de } \\
\text { la tarea de temblor, solo existieron } \\
\text { pequeñas diferencias antes, que se } \\
\text { incrementaron después de conocerlo, } \\
\text { favoreciendo al grupo sugestionable. }\end{array}$ \\
\hline
\end{tabular}

${ }^{1}$ Los autores no comparten el concepto de hipnosis como estado; no obstante, se mantiene respetando el texto original (-hypnosis state-). 


\section{Franquelo et al.}

\begin{tabular}{cc}
\hline Estudio & Objetivo \\
\hline & \\
Levitt y & Explorar los efectos de distintos \\
Brady & "estados" sobre la resistencia \\
$(1964)$ & muscular.
\end{tabular}

Muestra y diseño

8 enfermeras distribuidas

en tres grupos: hipnosis,

hipnosis + analgesia

hipnótica, vigilia +

instrucciones

motivacionales +

recompensa económica.

24 estudiantes divididos en

cuatro grupos: (a) hipnosis

+ instrucciones

motivacionales, (b)

hipnosis profunda + instrucciones

motivacionales, (c) instrucciones

motivacionales, y (d) control.

y Marks y las instrucciones motivacionales

(1967) sobre el aprendizaje kinestésico.
Resumen de los principales hallazgos

No se encontraron diferencias significativas entre los “estados". La interacción entre los "estados" y el orden de presentación sí resultó significativa, pero se atribuyó a la variabilidad intersujeto.
Las condiciones experimentales no afectaron al aprendizaje kinestésico, llegando a encontrarse que la hipnosis y las instrucciones motivacionales podían interferir con el aprendizaje de la tarea.

\begin{tabular}{|c|c|c|}
\hline $\begin{array}{c}\text { Fehr y Stern } \\
\text { (1967) }\end{array}$ & $\begin{array}{c}\text { Evaluar los efectos de la hipnosis } \\
\text { sobre estímulos relevantes e } \\
\text { irrelevantes. }\end{array}$ & $\begin{array}{l}24 \text { estudiantes divididas en } \\
\text { grupo experimental y grupo } \\
\text { control. }\end{array}$ \\
\hline
\end{tabular}

El grupo experimental mostró un incremento notable en el tiempo de reacción en comparación con el grupo control, así como una latencia de respuesta más larga frente a condiciones con distintos estímulos.

El rendimiento de todos los sujetos bajo estrés mejoró después de que fueran

Examinar los efectos de hipnosis, instrucciones de exhortación e instrucciones para la tarea sobre el London et rendimiento de individuos que al. (1968) difieren en su nivel de sugestionabilidad hipnótica en tareas bajo condiciones normales y con estrés inducido.
40 estudiantes divididos en dos grupos según su grado de sugestionabilidad.
Determinar si sugestiones hipnóticas positivas o sugestiones positivas sin hipnosis pueden ser utilizadas para mejorar el Arnold aprendizaje motor fino y grueso, y (1971) determinar si la hipnotizabilidad de los sujetos hace que afecten de forma distinta las sugestiones positivas cuando aprenden habilidades motoras.

Testar la hipótesis de que la mejora que tiene lugar en los test de habilidad en sujetos bajo hipnosis

Rader es el resultado de las condiciones
motivadoras implícitas en el (1972) procedimiento de inducción hipnótica más que del resultado de la mejora del rendimiento por el "estado" hipnótico.

hipnotizados o exhortados a rendir mejor; la exhortación no tuvo efectos significativos (solo efectos ligeros). El rendimiento del grupo poco sugestionable no fue significativamente superior al grupo sugestionable, aunque el rendimiento de este pareció más afectado por la hipnosis y la exhortación que el rendimiento de los poco sugestionables.

60 sujetos divididos en cuatro grupos (I) altamente hipnotizables en vigilia sin sugestiones (control), (II) sujetos altamente hipnotizables en vigilia con sugestiones positivas y de implicación, (III) sujetos altamente hipnotizables con sugestiones positivas y de implicación, (IIII) sujetos no hipnotizables en vigilia con sugestiones positivas y de implicación.

60 estudiantes asignados a cuatro condiciones experimentales: inducción hipnótica + instrucciones motivacionales, inducción hipnótica sin instrucciones motivacionales, instrucciones motivacionales y no hipnosis no instrucciones motivacionales.
No se encontraron diferencias significativas entre los grupos, según hipotetizaron los autores, debido al alto interés que todos los sujetos presentaron, considerándose la motivación intrínseca mayor que cualquier factor extrínseco proporcionado por el hipnotizador.

Los tiempos de reacción entre el grupo de instrucciones motivacionales e hipnosis + instrucciones motivacionales no fueron significativamente diferentes, pero ambos tuvieron mejores resultados que el grupo control, y este tuvo resultados significativamente mejores que el grupo de hipnosis sin motivación. 


\section{Hipnosis en Psicología del Deporte}

\begin{tabular}{cc}
\hline Estudio & Objetivo \\
\hline & \\
& \\
Morgan et & $\begin{array}{c}\text { Evaluar la influencia de sugestiones } \\
\text { hipnóticas sobre la percepción y } \\
\text { respuestas metabólicas a una tarea } \\
\text { de ergómetro de bicicleta. }\end{array}$
\end{tabular}

Muestra y diseño

Resumen de los principales hallazgos

Las sugestiones hipnóticas de trabajo duro evocaban percepciones de esfuerzo significativamente mayores que las sugestiones de trabajo ligero. No hubo diferencias significativas entre las percepciones de esfuerzo después de la hipnosis y la sugestión de trabajo moderado en vigilia. El ritmo cardíaco varió significativamente para las sugestiones de trabajo ligero y duro,

5 adultos con experiencia en investigaciones de laboratorio que impliquen esfuerzo muscular.
Albert y

Williams

(1975)

Jackson et al., (1979)
Examinar los efectos de sugestiones post-hipnóticas sobre la resistencia,

la escala Borg de esfuerzo percibido y la frecuencia cardíaca.
20 estudiantes distribuidos en grupo experimental (hipnosis) y grupo control. aunque la absorción de oxígeno no varió. Durante las sugestiones de trabajo ligero y moderado no se alcanzó estabilidad hasta el quinto minuto, mientras que en vigilia se alcanzó en el segundo minuto. No se encontraron diferencias entre los tratamientos ni sugestiones para la deuda de oxígeno, el volumen respiratorio por minuto, eficiencia respiratoria ni exceso de dióxido de carbono.

El tiempo de resistencia se reducía cuando se daban sugestiones de fatiga a los sujetos del grupo experimental, pero las sugestiones facilitadoras no mejoraron el rendimiento. La escala Borg refleja cambios subjetivos en los participantes según las sugestiones del hipnotizador. Los sujetos del grupo control no muestran cambios.

Se observó un incremento significativo en la resistencia de los grupos de

55 universitarios asignados a cinco grupos: control, hipnosis, motivación, baja

Explorar los efectos de distintos "estados" sobre la resistencia física en sujetos entrenados. susceptibilidad a la hipnosis

+ instrucciones

motivacionales y alta

susceptibilidad a la hipnosis

+ instrucciones

motivacionales.

motivación y alta susceptibilidad a la

hipnosis + motivación; la ventilación máxima se incrementó significativamente en el grupo con alta susceptibilidad en comparación con el grupo control, y también se dieron altas concentraciones de lactato en comparación con el grupo de hipnosis y baja susceptibilidad + motivación.

Investigar el beneficio de técnicas hipnóticas, de relajación y ensayo

$\begin{array}{ll}\text { Nelson } & \text { mental para la mejora del } \\ \text { (1980) } & \text { rendimiento en golfistas y }\end{array}$

$\begin{array}{ll}\text { Nelson } & \text { mental para la mejora del } \\ \text { (1980) } & \text { rendimiento en golfistas y }\end{array}$ corredores. Determinar los efectos de dos programas de entrenamiento Greer y (relajación progresiva e hipnosis)

Engs (1986) sobre la habilidad de estudiantes de tenis para aprender técnicas básicas.
41 golfistas y 50 corredores.

\section{0 principiantes en clases} de tenis asignados igualitariamente a una entre tres condiciones (relajación progresiva, hipnosis y control).

32 levantadores de pesos asignados aleatoriamente a cuatro tipos de tratamiento (CHI, CR, HO y control) con medidas pretest postest.
No se obtuvieron resultados significativos en ninguno de los dos deportes.

La relajación progresiva y la hipnosis junto a las instrucciones de práctica mental o visualización no resultaron más eficaces que las instrucciones tradicionales con explicación y demostración.

Examinar la eficacia de un enfoque cognitivo - hipnótico en imaginería

Howard y CHI), reestructuración cognitiva

(1986)
El grupo con CHI mostró diferencias estadísticamente significativas en todas las variables del pretest al postest. rendimiento neuromuscular, crecimiento muscular, reducción de ansiedad y mejora del autoconcepto. 


\section{Franquelo et al.}

\begin{tabular}{|c|c|c|c|}
\hline Estudio & Objetivo & Muestra y diseño & Resumen de los principales hallazgos \\
\hline $\begin{array}{l}\text { Winter } \\
(1986)\end{array}$ & $\begin{array}{c}\text { Combinar hipnosis con otras } \\
\text { técnicas con el objetivo de mejorar } \\
\text { marca personal (1) y de aumentar el } \\
\text { porcentaje de éxito en lanzamientos } \\
\text { libres }(2) .\end{array}$ & $\begin{array}{l}\text { Dos diseños de sujeto } \\
\text { único: saltador de pértiga } \\
\text { (1) y jugadora de } \\
\text { baloncesto (2). }\end{array}$ & $\begin{array}{l}\text { Los deportistas consiguieron mejorar su } \\
\text { rendimiento (mejores marcas personales } \\
\text { para el saltador de pértiga y aumento del } \\
23 \% \text { en el porcentaje de éxito en } \\
\text { lanzamientos libres para la jugadora de } \\
\text { baloncesto) y se sintieron con más } \\
\text { confianza. }\end{array}$ \\
\hline $\begin{array}{l}\text { Schreiber } \\
(1991)\end{array}$ & $\begin{array}{l}\text { Examinar si la hipnosis puede } \\
\text { mejorar el rendimiento de tiro en } \\
\text { baloncesto. }\end{array}$ & $\begin{array}{l}12 \text { jugadores y } 12 \text { jugadoras } \\
\text { de baloncesto asignados } \\
\text { aleatoriamente al grupo de } \\
\text { hipnosis y al grupo control. }\end{array}$ & $\begin{array}{l}\text { Los grupos del grupo de hipnosis } \\
\text { mostraron un mayor número acumulado } \\
\text { de puntos que el grupo control. }\end{array}$ \\
\hline $\begin{array}{l}\text { Robazza y } \\
\text { Bortoli } \\
\text { (1995) }\end{array}$ & $\begin{array}{l}\text { Combinar hipnosis activo - alerta } \\
\text { con hipnosis tradicional para } \\
\text { incrementar el rendimiento de tiro } \\
\text { en un arquero. }\end{array}$ & $\begin{array}{l}\text { Diseño de sujeto único (un } \\
\text { arquero). }\end{array}$ & $\begin{array}{l}\text { Los resultados demostraron un } \\
\text { incremento en el rendimiento en el tiro. }\end{array}$ \\
\hline $\begin{array}{l}\text { Pates y } \\
\text { Maynard } \\
(2000)\end{array}$ & $\begin{array}{l}\text { Examinar los efectos de una } \\
\text { intervención hipnótica sobre el } \\
\text { estado de flow y sobre el } \\
\text { rendimiento en el chip de golf. }\end{array}$ & $\begin{array}{l}3 \text { jugadores de golf con un } \\
\text { diseño ideográfico ABA de } \\
\text { sujeto único. }\end{array}$ & $\begin{array}{l}\text { Se encontró un aumento sobre el } \\
\text { rendimiento en el chip de golf; los } \\
\text { participantes se sintieron confiados, } \\
\text { relajados y con la situación bajo control; } \\
\text { así como } 2 \text { de los participantes } \\
\text { experimentaron un incremento en el } \\
\text { estado de flow. }\end{array}$ \\
\hline $\begin{array}{l}\text { Pates et al., } \\
\text { (2001a) }\end{array}$ & $\begin{array}{l}\text { Investigar los efectos de la hipnosis } \\
\text { sobre el rendimiento en el set and } \\
\text { jump. }\end{array}$ & $\begin{array}{l}3 \text { jugadores de baloncesto } \\
\text { universitarios con un diseño } \\
\text { de sujeto único ABA. }\end{array}$ & $\begin{array}{l}\text { Los resultados mostraron que los } \\
\text { participantes incrementaron su } \\
\text { rendimiento durante la intervención, } \\
\text { volviendo a la línea base al término de } \\
\text { esta, así como las sensaciones asociadas a } \\
\text { su rendimiento máximo. }\end{array}$ \\
\hline $\begin{array}{l}\text { Pates et al., } \\
\text { (2001b) }\end{array}$ & $\begin{array}{c}\text { Examinar los efectos de la hipnosis } \\
\text { sobre el rendimiento en golf y sobre } \\
\text { el estado de flow. }\end{array}$ & $\begin{array}{c}5 \text { jugadores de golf con } \\
\text { diseño ideográfico de sujeto } \\
\text { único. }\end{array}$ & $\begin{array}{c}\text { Se encontró que los participantes } \\
\text { incrementaron su rendimiento y su } \\
\text { percepción sobre las sensaciones } \\
\text { asociadas a los estados de flow. }\end{array}$ \\
\hline $\begin{array}{l}\text { Pates et al., } \\
\quad(2002)\end{array}$ & $\begin{array}{l}\text { Examinar los efectos de la hipnosis } \\
\text { sobre los estados de flow y sobre el } \\
\text { rendimiento en triples. }\end{array}$ & $\begin{array}{l}5 \text { jugadores de baloncesto } \\
\text { universitarios con un diseño } \\
\text { ideográfico de sujeto único. }\end{array}$ & $\begin{array}{l}\text { Los resultados indican que los cinco } \\
\text { jugadores mejoraron su rendimiento en } \\
\text { triples, así como su estado de flow con } \\
\text { respecto a las medidas de base. }\end{array}$ \\
\hline $\begin{array}{l}\text { Pates y } \\
\text { Palmi } \\
(2002)\end{array}$ & $\begin{array}{l}\text { Examinar los efectos de la hipnosis } \\
\text { sobre los estados de flow y sobre el } \\
\text { servicio corto en bádminton. }\end{array}$ & $\begin{array}{l}4 \text { jugadoras competitivas de } \\
\text { bádminton con diseño } \\
\text { ideográfico de diseño } \\
\text { único. }\end{array}$ & $\begin{array}{c}\text { Los resultados indican que las jugadoras } \\
\text { aumentaron el rendimiento en el servicio } \\
\text { corto, aumentaron sus puntuaciones en } \\
\text { flow y se sintieron más tranquilas, } \\
\text { relajadas, decididas, felices y } \\
\text { concentradas. }\end{array}$ \\
\hline $\begin{array}{l}\text { Morton } \\
(2003)\end{array}$ & $\begin{array}{l}\text { Describir múltiples usos de auto- } \\
\text { hipnosis para hacer frente a } \\
\text { distintos desafíos en escalada. }\end{array}$ & $\begin{array}{l}\text { Diseño de caso único: } \\
\text { escaladora. }\end{array}$ & $\begin{array}{l}\text { Se produjo una mejora de la habilidad } \\
\text { para manejar y reducir la ansiedad, así } \\
\text { como para aproximarse al a zona de } \\
\text { funcionamiento óptimo. Se consiguió una } \\
\text { estrategia efectiva para dirigir y organizar } \\
\text { la energía, y se consiguió eliminar } \\
\text { pesadillas y miedos recurrentes. Se } \\
\text { produjo un aumento en la autoconfianza. }\end{array}$ \\
\hline $\begin{array}{l}\text { Fernández et } \\
\text { al., (2004) }\end{array}$ & $\begin{array}{l}\text { Evaluar la eficacia diferencial de } \\
\text { una intervención con hipnosis y } \\
\text { ACT para la mejora de la fuerza } \\
\text { física en piragüistas. }\end{array}$ & $\begin{array}{l}16 \text { practicantes de } \\
\text { piragüismo distribuidos al } \\
\text { azar en dos grupos de } \\
\text { tratamiento (hipnosis y } \\
\text { ACT). }\end{array}$ & $\begin{array}{l}\text { Se observó un aumento en el número de } \\
\text { repeticiones en remo inclinado. }\end{array}$ \\
\hline $\begin{array}{l}\text { Lindsay et } \\
\text { al., (2005) }\end{array}$ & $\begin{array}{c}\text { Evaluar la eficacia de una } \\
\text { intervención hipnótica sobre los } \\
\text { estados de flow y el rendimiento en } \\
\text { ciclismo competitivo. }\end{array}$ & $\begin{array}{l}\text { Diseño de línea base } \\
\text { múltiple con sujeto único (3 } \\
\text { ciclistas). }\end{array}$ & $\begin{array}{l}\text { Los resultados obtenidos sugieren una } \\
\text { mejora del rendimiento en competición y } \\
\text { un incremento de los sentimientos y } \\
\text { cogniciones de flow. }\end{array}$ \\
\hline $\begin{array}{l}\text { Newmark y } \\
\text { Bogacki } \\
(2005)\end{array}$ & $\begin{array}{l}\text { Realizar intervenciones hipnóticas } \\
\text { ante demanda en distintos casos } \\
\text { con objetivos de mejora de } \\
\text { rendimiento. }\end{array}$ & $\begin{array}{l}\text { Cuatro diseños de caso } \\
\text { único: un futbolista (1), un } \\
\text { jugador de baloncesto (2), } \\
\text { un golfista amateur (3) y un } \\
\text { futbolista (4). }\end{array}$ & $\begin{array}{l}\text { Se produjeron mejoras del rendimiento } \\
\text { en todos los casos. Además, resultó útil } \\
\text { en la intervención para depresión (1) y } \\
\text { control de la ira (4). }\end{array}$ \\
\hline
\end{tabular}




\begin{tabular}{|c|c|c|c|}
\hline \multicolumn{4}{|c|}{ Hipnosis en Psicología del Deporte } \\
\hline Estudio & Objetivo & Muestra y diseño & Resumen de los principales hallazgos \\
\hline $\begin{array}{l}\text { Anchique } \\
(2006)\end{array}$ & $\begin{array}{c}\text { Evaluar la utilidad de la } \\
\text { autosugestión positiva dirigida al } \\
\text { incremento en la efectividad de } \\
\text { primer y segundo servicio en } \\
\text { jugadores de tenis. }\end{array}$ & $\begin{array}{l}9 \text { tenistas con diseño } \mathrm{O} 1 \mathrm{X} \\
\mathrm{O} 2 \text { y medidas pretest - } \\
\text { postest. }\end{array}$ & $\begin{array}{c}\text { No se encontraron diferencias en ambas } \\
\text { mediciones entre los valores del primer y } \\
\text { el segundo servicio, pero se reportaron } \\
\text { diferencias significativas a favor de la } \\
\text { efectividad en la totalidad de los } \\
\text { servicios y en la disminución de las } \\
\text { dobles faltas cometidas. }\end{array}$ \\
\hline $\begin{array}{c}\text { Barker y } \\
\text { Jones (2006) }\end{array}$ & $\begin{array}{c}\text { Evaluar el impacto de la hipnosis, } \\
\text { el perfeccionamiento y el } \\
\text { automodelado en el incremento de } \\
\text { la autoeficacia en jugadores de } \\
\text { cricket. }\end{array}$ & $\begin{array}{l}\text { Diseño de sujeto único } \\
\text { (jugador de cricket semi - } \\
\text { profesional) con medidas } \\
\text { pretest - postest. }\end{array}$ & $\begin{array}{l}\text { Los resultados indican diferencias } \\
\text { significativas en los niveles de } \\
\text { autoeficacia antes y después de la } \\
\text { intervención, así como un incremento del } \\
\text { rendimiento. }\end{array}$ \\
\hline $\begin{array}{l}\text { Cracium y } \\
\text { Szatmari } \\
(2007)\end{array}$ & $\begin{array}{l}\text { Examinar los efectos de la hipnosis } \\
\text { activo-alerta sobre los estados de } \\
\text { ánimo de deportistas de élite. }\end{array}$ & $\begin{array}{l}12 \text { deportistas de élite } \\
\text { (equipo olímpico femenino } \\
\text { de judo). }\end{array}$ & $\begin{array}{l}\text { Se produjo un cambio destacado en los } \\
\text { estados anímicos de todas las deportistas, } \\
\text { presentando un perfil de tipo iceberg tras } \\
\text { la sesión de hipnosis. }\end{array}$ \\
\hline
\end{tabular}

Explorar la eficacia de la hipnosis como intervención para reducir el afecto negativo y aumentar la autoeficacia, la confianza, el afecto positivo y el rendimiento; aumentar la base de conocimiento sobre la Barker y eficacia de las habilidades en Jones (2008) escenarios de la vida real y en el fútbol profesional; investigar más a fondo la relación entre la autoeficacia y los afectos; $y$ examinar las cuestiones aplicadas al uso de la hipnosis en el fútbol profesional.

Diseño de sujeto único (jugador de fútbol profesional) con medidas pretest - postest.
Los resultados sugieren que la hipnosis puede ser utilizada para la mejora de la autoeficacia, los afectos y el rendimiento deportivo.

\begin{tabular}{cc} 
& profesional. \\
\hline Barker et al., & Evaluar los efectos de la hipnosis \\
(2010) & sobre la autoeficacia y el \\
& rendimiento en fútbol.
\end{tabular}

59 jugadores universitarios de soccer wall-volley distribuidos aleatoriamente en un grupo de hipnosis (n $=30$ ) y un grupo control ( $\mathrm{n}$ $=29$ ) con medidas pretest postest.

24 practicantes de ciclismo distribuidos aleatoriamente a un grupo experimental con hipnosis y a un grupo control con medidas pretest - postest.

Evaluar la eficacia de la hipnosis en Fernández et la modificación de un conjunto de al., (2013) durante la realización de pruebas de esfuerzo.
24 ciclistas distribuidos aleatoriamente a un grupo experimental con hipnosis y

a un grupo control con medidas pretest - postest.

Los resultados indican que el grupo de hipnosis fue más eficaz y rindió mejor que el grupo control. Además, a pesar de que los cambios en autoeficacia se asociaron a camios en el rendimiento, los efectos de la hipnosis no estuvieron mediados por los cambios en autoeficacia.

Los resultados indican una relación estadísticamente significativa entre la resistencia al esfuerzo y el tipo de

tratamiento, indicando que la resistencia al esfuerzo mejoraba conforme se aumentaba el número de sesiones.

No se encontraron diferencias estadísticamente significativas entre los grupos en las variables, pero sí se observó una tendencia en el grupo experimental a puntuar más positivamente en las variables psicológicas.

Diseño de sujeto único $\mathrm{AB}$ (un jugador de golf profesional).
Los resultados indican que el promedio de golpes y el promedio de puntos incrementó tras la intervención.
Pates (2013) intervención con hipnosis sobre

flow en jugadores de golf profesional.

Pates y Examinar los efectos de la hipnosis

Cowen sobre el rendimiento y el estado de

(2013) flow.
Diseño de sujeto único $\mathrm{AB}$ (golfista).
Los resultados indican que la intervención mejoró consistentemente el rendimiento y la intensidad de la experiencia de flow del participante durante las competiciones. 


\section{Franquelo et al.}

\begin{tabular}{|c|c|c|c|}
\hline Estudio & Objetivo & Muestra y diseño & Resumen de los principales hallazgos \\
\hline $\begin{array}{c}\text { Jalene y } \\
\text { Wulf (2014) }\end{array}$ & $\begin{array}{l}\text { Examinar si el aprendizaje de } \\
\text { habilidades motoras puede ser } \\
\text { mejorado a través de una } \\
\text { intervención con hipnosis. }\end{array}$ & $\begin{array}{l}22 \text { estudiantes divididos en } \\
\text { grupo control y grupo } \\
\text { experimental. }\end{array}$ & $\begin{array}{l}\text { El grupo de hipnosis mostró un aumento } \\
\text { en la precisión de los lanzamientos en } \\
\text { relación al pretest y un rendimiento más } \\
\text { eficaz que el grupo control, que presentó } \\
\text { un rendimiento similar al pretest. }\end{array}$ \\
\hline Pates (2014) & $\begin{array}{l}\text { Examinar los efectos de una } \\
\text { intervención con hipnosis sobre el } \\
\text { rendimiento y la experiencia de } \\
\text { flow. }\end{array}$ & $\begin{array}{l}\text { Diseño de sujeto único AB } \\
\text { (jugador de golf } \\
\text { profesional). }\end{array}$ & $\begin{array}{l}\text { Los resultados mostraron un aumento en } \\
\text { el promedio de golpes y en la media de } \\
\text { las puntuaciones de flow. Además, los } \\
\text { datos revelan que la hipnosis afectó } \\
\text { positivamente emociones, pensamientos, } \\
\text { sentimientos y percepciones. }\end{array}$ \\
\hline $\begin{array}{l}\text { Zurita et al., } \\
\text { (2014) }\end{array}$ & $\begin{array}{l}\text { Comprobar la eficacia de un } \\
\text { programa de hipnosis para la } \\
\text { mejora de la fuerza física. }\end{array}$ & $\begin{array}{l}\text { Diseño de sujeto único (un } \\
\text { ciclista) con medidas } \\
\text { pretest - postest. }\end{array}$ & $\begin{array}{l}\text { Se encontró una mejora de la fuerza (que } \\
\text { se mantiene durante los siguientes } 6 \\
\text { meses), mientras la percepción subjetiva } \\
\text { de fatiga se mantuvo constante. }\end{array}$ \\
\hline $\begin{array}{l}\text { Hernández- } \\
\text { Mendo et } \\
\text { al., (2017) }\end{array}$ & $\begin{array}{l}\text { Realizar una intervención hipnótica } \\
\text { programada como respuesta a } \\
\text { solicitudes de intervención (tras } \\
\text { accidente automovilístico (1), } \\
\text { lesión (2), trastorno en conducta } \\
\text { alimentaria (3) y para mejora del } \\
\text { rendimiento (4). }\end{array}$ & $\begin{array}{l}\text { Cuatro diseños de sujeto } \\
\text { único: corredor de atletismo } \\
\text { (1), saltadora de pértiga (2), } \\
\text { corredora de montaña y } \\
\text { ultra-tail (3) y futbolista } \\
\text { (4). }\end{array}$ & $\begin{array}{l}\text { (1) Se redujo el dolor y los días de } \\
\text { reposo, permitiendo reanudar antes de lo } \\
\text { previsto los entrenamientos y se } \\
\text { restableció el nivel de ejecución anterior. } \\
\text { (2) Se obtuvieron resultados } \\
\text { satisfactorios según las declaraciones de } \\
\text { la saltadora, que se reflejaron en un claro } \\
\text { aumento en las distancias de salto. (3) Se } \\
\text { eliminaron los indicios de ansiedad y la } \\
\text { provocación de vómito, y se eliminó la } \\
\text { imposibilidad de beber agua (además, se } \\
\text { consiguieron muy buenos resultados en } \\
\text { competición). (4) Se consiguió un } \\
\text { aumento del rendimiento. }\end{array}$ \\
\hline Pates & $\begin{array}{l}\text { Investigar los efectos de una } \\
\text { intervención basada en hipnosis } \\
\text { sobre el rendimiento y sobre un } \\
\text { estado mental denominado } \\
\text { "Clutch". }\end{array}$ & $\begin{array}{c}\text { Tres golfistas profesionales } \\
\text { con diseño de caso único } \\
\text { AB. }\end{array}$ & $\begin{array}{l}\text { Los resultados indican que la hipnosis } \\
\text { puede mejorar consistentemente el } \\
\text { rendimiento, así como incrementar las } \\
\text { sensaciones asociadas con el estado } \\
\text { "Clutch". }\end{array}$ \\
\hline $\begin{array}{l}\text { Mattle et al., } \\
\quad(2020)\end{array}$ & $\begin{array}{l}\text { Investigar los efectos de una } \\
\text { intervención de hipnosis en el } \\
\text { rendimiento de tiro. }\end{array}$ & $\begin{array}{c}8 \text { atletas miembros del } \\
\text { equipo nacional con diseño } \\
\text { de caso único ABA con dos } \\
\text { medidas base. }\end{array}$ & $\begin{array}{l}\text { En tres atletas se produjo un pequeño } \\
\text { efecto positivo en su rendimiento en } \\
\text { comparación con el resto de las medidas; } \\
\text { seis sujetos tuvieron pequeñas mejoras en } \\
\text { comparación con una de las medidas } \\
\text { base; y dos sujetos tuvieron un } \\
\text { decremento en su rendimiento. Los } \\
\text { atletas, además, afirmaron sentirse más } \\
\text { tranquilos, más concentrados y seguro de } \\
\text { sí mismos al comienzo de la competición. }\end{array}$ \\
\hline
\end{tabular}

\section{Consideraciones metodológicas}

Los 45 estudios incluidos en esta revisión responden a un acercamiento relativo a cinco criterios metodológicos mencionados previamente: (a) especificación de la muestra, (b) uso de la hipnosis como técnica, (c) medición de la sugestionabilidad hipnótica de los participantes, (d) resultados estadísticos directos sobre los efectos del uso de la hipnosis, y (e) medida del tamaño del efecto. Algunos de estos criterios han sido seleccionados por responder directamente a los objetivos del estudio, y otros por reflejar un alto rigor metodológico. La Tabla 2 evalúa los 45 estudios incluidos en base a los criterios metodológicos establecidos, designando con "Sí" o "No" el cumplimiento de estos.

Tabla 2. Cumplimiento de los criterios metodológicos. 


\section{Hipnosis en Psicología del Deporte}

\begin{tabular}{|c|c|c|c|c|c|}
\hline Estudio & $\begin{array}{l}\text { Especificación } \\
\text { de la muestra }\end{array}$ & $\begin{array}{l}\text { Uso de la } \\
\text { hipnosis } \\
\text { como técnica }\end{array}$ & $\begin{array}{c}\text { Medición de la } \\
\text { sugestionabilidad }\end{array}$ & $\begin{array}{c}\text { Resultados } \\
\text { estadísticos sobre los } \\
\text { efectos del uso de la } \\
\text { hipnosis }\end{array}$ & $\begin{array}{l}\text { Medida del } \\
\text { tamaño del } \\
\text { efecto }\end{array}$ \\
\hline Young (1925) & No & Sí & No & Sí & No \\
\hline Roush (1951) & Sí & Sí & No & Sí & No \\
\hline Orne (1959) & No & Sí & Sí & Sí & No \\
\hline London y Fuhrer (1961) & Sí & Sí & Sí & Sí & No \\
\hline Rosenhan y London (1963) & No & Sí & Sí & Sí & No \\
\hline Levitt y Brady (1964) & No & Sí & Sí & Sí & No \\
\hline Edmonston y Marks (1967) & No & Sí & Sí & Sí & No \\
\hline Fehr y Stern (1967) & No & Sí & Sí & Sí & No \\
\hline London et al., (1968) & No & Sí & Sí & Sí & No \\
\hline Arnold (1971) & No & Sí & Sí & Sí & No \\
\hline Rader (1972) & Sí & Sí & No & Sí & No \\
\hline Morgan et al., (1973) & Sí & Sí & Sí & Sí & No \\
\hline Albert y Williams (1975) & Sí & Sí & Sí & Sí & No \\
\hline Jackson et al., (1979) & No & Sí & Sí & Sí & No \\
\hline Nelson (1980) & Sí & Sí & No & Sí & No \\
\hline Greer y Engs (1986) & No & Sí & No & Sí & No \\
\hline Howard y Reardon (1986) & Sí & Sí & $\mathrm{No}^{*}$ & Sí & No \\
\hline Winter (1986) & Sí & Sí & No & No & No \\
\hline Schreiber (1991) & Sí & Sí & No & Sí & No \\
\hline Robazza y Bortoli (1995) & Sí & Sí & No & Sí & No \\
\hline Pates y Maynard (2000) & Sí & $\mathrm{Si}$ & No & Sí & No \\
\hline Pates et al., (2001a) & Sí & Sí & No & Sí & No \\
\hline Pates et al., (2001b) & Sí & Sí & No & Sí & No \\
\hline Pates et al., (2002) & Sí & Sí & No & Sí & No \\
\hline Pates y Palmi (2002) & Sí & Sí & No & Sí & No \\
\hline Morton (2003) & No & Sí & No & No & No \\
\hline Fernández et al., (2004) & Sí & Sí & Sí & Sí & No \\
\hline Lindsay et al., (2005) & Sí & Sí & No & Sí & No \\
\hline Newmark y Bogacki (2005) & Sí & Sí & No & No & No \\
\hline Anchique (2006) & Sí & No & No & No & No \\
\hline Barker y Jones (2006) & Sí & Sí & No* & Sí & No \\
\hline Cracium y Szatmari (2007) & No & Sí & No & Sí & No \\
\hline Barker y Jones (2008) & Sí & Sí & No* & Sí & No \\
\hline Barker et al., (2010) & Sí & Sí & $\mathrm{No}^{*}$ & Sí & Sí \\
\hline Fernández et al., (2012) & Sí & Sí & Sí & Sí & Sí \\
\hline Fernández et al., (2013) & Sí & Sí & Sí & Sí & Sí \\
\hline Pates (2013) & Sí & Sí & No & Sí & No \\
\hline Pates y Cowen (2013) & Sí & Sí & No & Sí & No \\
\hline Jalene y Wulf (2014) & Sí & Sí & Sí & Sí & No \\
\hline Pates (2014) & Sí & Sí & No & Sí & No \\
\hline Zurita et al., (2014) & Sí & Sí & Sí & Sí & No \\
\hline Hernández-Mendo et al., (2017) & Sí & Sí & Sí & Sí & No \\
\hline Pates (2019) & Sí & Sí & No & Sí & No \\
\hline Mattle et al., (2020) & Sí & Sí & No & No & No \\
\hline
\end{tabular}

Notas: * no miden la sugestionabilidad, pero sí hacen una introducción a la técnica, aportando información y permitiendo al participante realizar preguntas para reducir mitos y concepciones erróneas sobre la hipnosis y para favorecer el establecimiento del rapport.

Especificación de la muestra 


\section{Franquelo et al.}

Se valorará que se especifique la edad y sexo de los participantes, con el objetivo de poder determinar la población a la que el estudio puede generalizarse. Todos los estudios, a excepción de Young (1925), Orne (1959), London et al., (1968), Arnold (1971) y Greer y Engs (1986) (que no hacen especificaciones de la muestra); y Rosenhan y London (1963), Levitt y Brady (1964), Edmonston y Marks (1967), Fehr y Stern (1967), Jackson et al., (1979), Morton (2003) y Cracium y Szatmari (2007) (que solo especifican el sexo), añaden una descripción detallada de la muestra utilizada.

\section{Uso de la hipnosis como técnica}

Respondiendo a los objetivos del estudio, será necesario que aquellos estudiados incorporen la técnica de la hipnosis en el tratamiento o intervención con los participantes, a fin de explorar su eficacia. Todos los estudios incluidos, a excepción de Anchique (2006), que emplea autosugestión positiva como técnica, emplean la hipnosis como tratamiento o intervención de los (o algunos de los) participantes.

\section{Medición de la sugestionabilidad}

La sugestionabilidad hipnótica hace referencia a la tendencia personal a dar respuesta a una sugestión hipnótica (Gur, 1978). Puede ser evaluada a través de escalas estandarizadas que consisten en inducciones hipnóticas con un conjunto de pruebas de sugestión (Gur, 1978). Distintos autores, como Lynn y Shindler (2002) recomiendan una evaluación de la sugestionabilidad hipnótica para todos aquellos estudios en los que se pretenda evaluar los efectos de la hipnosis como tratamiento o intervención, ya que esto permitirá evaluar si existen resultados significativos y cómo estos varían en función a los niveles de sugestionabilidad que presenten los participantes. Por el contrario, otros autores (p. e. Montgomery et al., 2011) afirman que la sugestionabilidad hipnótica es irrelevante, explicando un porcentaje muy pequeño ( $6 \%$ de la varianza) de los resultados en estudios clínicos, aunque significativo. Incluso se podría obtener una mejor respuesta a la intervención hipnótica si no se pasa por la experiencia de evaluación de la sugestionabilidad con las escalas al uso, pues incluyen ítems muy difíciles que implican fracaso para a la mayoría de usurarios lo que podría ser un factor desmotivador (Montgomery et al., 2011). Diecisiete de los estudios incluidos añadieron una medición de la sugestionabilidad hipnótica previa a la intervención o tratamiento con hipnosis. Del resto de estudios, cuatro, a pesar de no medir la sugestionabilidad de los participantes, incluyeron una breve introducción, con el fin de que los participantes conocieran la técnica y redujeran concepciones y mitos erróneos.

Resultados estadísticos sobre los efectos del uso de la hipnosis
Respondiendo a los objetivos del estudio, se necesita que se reporten, de forma estadística, los efectos de utilizar la hipnosis como técnica en la mejora del rendimiento. Es decir, se necesita una medida estadística de la eficacia de esta técnica en el deporte y el ejercicio físico, a fin de tener la posibilidad de clasificarla como eficaz con el suficiente apoyo empírico. Todos los estudios incluidos, a excepción de Winter (1986), Morton (2003), Newmark y Bogacki (2005), Anchique (2006) y Mattle et al., (2020), incluyeron resultados estadísticos sobre los resultados derivados del uso de la hipnosis, a pesar de que algunos solo reportaron resultados a nivel de estadísticos descriptivos.

\section{Medida del tamaño del efecto}

El tamaño del efecto tiene por objetivo cuantificar la magnitud de una relación o efecto (Cohen, 1988). Dicho de otro modo, permite inferir el grado de generalización que posee un determinado resultado estadístico en la población de la muestra estudiada, permitiendo así, conocer, en qué medida se puede esperar este fenómeno en la población (Ledesma et al., 2008). Estos valores, junto a los valores de probabilidad proporcionados, permiten interpretar de forma sustantiva los resultados obtenidos en una investigación (Frías-Navarro et al., 2000). Únicamente tres de los estudios incluidos presentaron una medida del tamaño del efecto en los resultados.

\section{DISCUSIÓN}

Los objetivos del estudio fueron realizar una revisión sistemática acerca de la eficacia de la hipnosis sobre el rendimiento en deportistas y practicantes de ejercicio físico, o sobre variables o respuestas que medien o afecten al rendimiento deportivo y/o la práctica físico - deportiva, describiendo los resultados de aquellos estudios que se centrasen en explorar estas variables, así como describir la fuerza metodológica de los mismos, atendiendo a una serie de criterios.

La revisión realizada, que contempló un total de 45 estudios, sugiere que cuando se emplea la hipnosis como técnica para la mejora del rendimiento deportivo o de variables asociadas, esta tiende a resultar más eficaz que otras técnicas utilizadas. Más concretamente, atendiendo a los artículos que trabajan con distintos tipos de deportes, se encuentra que en deportes individuales (Anchique, 2006; Cracium y Szatmari, 2007; Fernández et al., 2004; Fernández et al., 2012; Fernández et al., 2013; HernándezMendo et al., 2017; Howard y Reardon, 1986; Lindsay et al., 2005; Mattle et al., 2020; Morton, 2003; Newmark y Bogacki, 2005; Pates, 2013; Pates, 2014; Pates, 2019; Pates y Cowen, 2013; Pates y Maynard, 2000; Pates et al., 2001b; Pates y Palmi, 2002; Robazza y Bortoli, 1995; Winter, 1986; Zurita et al., 2014) se encuentran diferencias significativas en cuanto a eficacia con el uso de la hipnosis, encontrándose mejoras en el rendimiento de los deportistas $\mathrm{y}$ practicantes de actividad físico-deportiva, y se reportan 


\section{Hipnosis en Psicología del Deporte}

incrementos en el nivel de autoeficacia y de concentración. Además, también se encuentran mejoras en los estados de ánimo (incluso proporcionando beneficios en el tratamiento de la depresión), $\mathrm{y}$, en algunos casos, en variables físicas. Por otra parte, los resultados en deportes colectivos (Barker y Jones, 2006, 2008; Barker et al., 2010; Hernández-Mendo et al., 2017; Newmark y Bogacki, 2005; Pates et al., 2002; Pates et al., 2001a; Schreiber, 1991; Winter 1986) señalan un incremento en los niveles de autoeficacia de los deportistas y del rendimiento, manteniéndose estos efectos en el tiempo.

Por otra parte, atendiendo a los criterios metodológicos establecidos, se seleccionaron un total de cinco criterios, a saber: especificación de la muestra, uso de la hipnosis y medición de la sugestionabilidad, resultados estadísticos sobre los efectos de la hipnosis y medida del tamaño del efecto. Atendiendo a los estudios incluidos, la mayor parte de ellos responden a la totalidad de estos criterios, exceptuando dos, medición de la sugestionabilidad y la medida del tamaño del efecto. Con respecto al primero, los estudios no tienden a evaluar psicométricamente la sugestionabilidad de los participantes. Por otra parte, con respecto a la medida del tamaño del efecto, es la herramienta estadística que permite conocer en qué medida puede esperarse el efecto estudiado en la población de la que se extrae la muestra (Ledesma et al., 2008), de ahí se deriva su importancia. Además, en los últimos años, existe cierta actividad por parte de las asociaciones y revistas científicas para alentar a los investigadores a reportar esta medida en sus estudios, junto a la significación estadística (FríasNavarro et al., 2000). En esta línea, cabe destacar que algunos de los estudios incluidos, especialmente aquellos más antiguos, presentan algunas lagunas en cuanto a rigor metodológico o estadístico, siguiendo las recomendaciones de los autores más actuales. No obstante, hay que señalar la importancia teórica del contenido de estos artículos, que sientan las bases sobre la eficiencia y la eficacia de la hipnosis en la disciplina.

Por lo tanto, teniendo en cuenta los resultados obtenidos, resulta de vital importancia continuar contribuyendo al desarrollo del bagaje científico de la hipnosis en el campo de la intervención en Psicología del Deporte, vistas las ventajas descritas que esta técnica puede aportar en el ámbito. No son muchos, como se puede comprobar, los estudios disponibles que se centren en evaluar la eficacia de la hipnosis sobre el rendimiento en deportistas o practicantes de ejercicio físico (contrariamente a la literatura existente sobre la utilización en la disciplina, ampliamente avalada y justificada (Hernández-Mendo et al., 2017)). Cabe añadir, además, que entre las limitaciones del estudio se encuentra que el número de estudios disponibles se reduce atendiendo al objetivo del estudio (relación entre hipnosis y rendimiento deportivo o variables o respuestas que medien o afecten al rendimiento deportivo y/o la práctica físico - deportiva), pudiendo encontrar un mayor número de resultados si se atiende a otras técnicas, como imagery, o al uso de la hipnosis con otros fines (p. e. para intervenciones con fines terapéuticos $o$ bajo situaciones/condiciones médicas). Así mismo, en algunas de las investigaciones realizadas, como se destacaba anteriormente, no se cumplen con criterios metodológicos del todo estrictos. En definitiva, y como ya afirmaba Capafons (2012), resulta aún necesaria la realización de más investigaciones con mayores muestras y con diseños experimentales mejorados, que determinen la eficacia de la hipnosis en aquellas áreas donde la evidencia necesita ser, todavía, más respaldada.

\section{REFERENCIAS}

AAHEA (2017). Definiciones. Definiciones de hipnosis.

https://www.aahea.net/hipnosis/definiciones-dehipnosis/

Albert, I., y Williams, M. H. (1975). Effects of posthypnotic suggestions on muscular endurance. Perceptual and Motor Skills, 40, 131-139. https://doi.org/10.1080/00207146408409254

American Psychological Association (Society of Psychological Hypnosis) (2004). Division 30' new definition of hypnosis. Psychological Bulletin, 13, 13. https://doi.org/10.1037/e405982005-007

APA Division 30, Society of Psychological Hypnosis (2014). Definition and Description of Hypnosis. http://www.apadivisions.org/division-

30/about/index.aspx

Anchique, F. A. (2006). Efectos de la autosugestión positiva en el incremento de la efectividad del servicio en tenistas de campo. Revista Iberoamericana de Psicología del Ejercicio y el Deporte, 1(1), 13-26. https://doi.org/10.2307/j.ctv13vdf2r.9

Arnold, J. (1971). Effects of hypnosis on the learning of two motor skills. Research Quarterly, 42, 1-6. https://doi.org/10.1080/10671188.1971.10615027

Blankfield, R. P. (1991). Suggestion, relaxation, and hypnosis as adjuncts in the care of surgery patients: A review of the literature. American Journal of Clinical Hypnosis, 33, 1782-1786. https://doi.org/10.1080/00029157.1991.10402927

Barker, J. B., y Jones, M. V. (2006). Using Hypnosis, Technique Refinement, and Self-Modeling to Enhance Self-Efficacy: A Case Study in Cricket. The Sport 


\section{Franquelo et al.}

Psychologist, $\quad 20, \quad 94-110$. https://doi.org/10.1123/tsp.20.1.94

Barker, J. B., y Jones, M. V. (2008). The Effects of Hypnosis on Self-Efficacy, Affect, and Soccer Performance: A Case Study. Journal of Clinical Sport Psychology, 2, 127-147. https://doi.org/10.1123/jcsp.2.2.127

Barker, J., Jones, M., y Greenlees, I. (2010). Assessing the Immediate and Maintained Effects of Hypnosis on Self-Efficacy and Soccer Wall-Volley Performance. Journal of Sport \& Exercise Psychology, 32, 243-252. https://doi.org/10.1123/jsep.32.2.243

Barker, J., Jones, M., y Greenlees, I. (2013). Using Hypnosis to Enhance Self-Efficacy in Sport Performers. Journal of Clinical Sport Psychology, 7, 229-247. https://doi.org/10.1123/jcsp.7.3.228

Capafons, A. (2012). Hipnosis (2ªd.). Edt. Síntesis. Madrid

Chambless, D. L., y Hollon, S. D. (1998). Defining empirically supported therapies. Journal of Consulting and Clinical Psychology, 66, 7-18. https://doi.org/10.1037/0022-006x.66.1.7

Cohen, J. (1988). Statistical power analysis for the behavioral science (2nd ed.). Hillsdale, NJ: Lawrence Erlbaum Associates.

Cracium, M., y Szatmari, C. (2007). Los efectos de la hipnosis activo-viva sobre el estado anímico de los deportistas de élite. Revista Iberoamericana de Psicología del Ejercicio y el Deporte, 1(2), 45-52. https://doi.org/10.5093/rpadef2020a1

Edmonston, W. E., y Marks, H. E. (1967). The effects of hypnosis and motivational instructions on kinesthetic learning. The American Journal of Clinical Hypnosis, 9 , 252-255. https://doi.org/10.1080/00029157.1967.10402560

Elkins, G., Marcus, J., Bates, J., Rajab, M. H., y Cook, T. (2006). Intensive hypnotherapy for smoking cessation: A prospective study. International Journal of Clinical and Experimental Hypnosis, 54, 303-315. https://doi.org/10.1080/00207140600689512

Elkins, G., y Rajab, M. H. (2004). Clinical hypnosis for smoking cessation: Preliminary results of a threesession intervention. International Journal of Clinical and Experimental Hypnosis, 52, 73-81. https://doi.org/10.1076/iceh.52.1.73.23921
Faymonville, M. E., Mambourg, P. H., Joris, J., Vrijens, B., Fissette, J., Albert, A., y Lamy, M. (1997). Psychological approaches during conscious sedation. Hypnosis versus stress reducing strategies: A prospective randomized study. Pain, 73, 361-367. https://doi.org/10.1016/s0304-3959(97)00122-x

Faymonville, M. E., Meurisse, M., y Fissette, J. (1999). Hypnosedation: A valuable alternative to traditional anaesthetic technicques. Acta Chirurgica Belgica, 99, 141-146. https://doi.org/10.1080/00015458.1999.12098466

Fehr, F. S., y Stem, J. A. (1967). The effect of hypnosis on attention to relevant and irrelevant stimuli. International Journal of Clinical and Experimental Hypnosis, $\quad 15, \quad 134-143$. https://doi.org/10.1080/00207146708407519

Fernández, R., Sánchez, L., y Zurita, F. (2013). Eficacia de la hipnosis en la modificación de variables psicológicas y fisiológicas en deportistas. Universitas Psychologica, 12(2), 483-491. https://doi.org/10.11144/javeriana.upsy12-2.ehmv

Fernández, R., Secades, R., Terrados, N., García, E., y García, J. M. (2004). Efecto de la hipnosis y la terapia de aceptación y compromiso (ACT) en la mejora de la fuerza física en piragüistas. International Journal of Clinical and Health Psychology, 4(3), 481-493. https://doi.org/10.5209/psic.63651

Fernández, R., Zurita, F., Sánchez, L, Linares, D., y Pérez, A. (2012). Influencia de la hipnosis en la resistencia al esfuerzo en ciclistas. Cultura, Ciencia y Deporte, $\quad 7(21)$,

191-199.

https://doi.org/10.12800/ccd.v7i21.84

Frías-Navarro, M. D., Pascual-Llobell, J., y GarcíaPérez, J. F. (2000). Tamaño del efecto del tratamiento y significación estadística. Psicothema, 12, 236-240.

Green, J. P. (1996). Cognitive-behavioral hypnotherapy for smoking cessation: A case study in a group setting. In S.J. Lynn, I. Kirsch, y J.W. Rhue (Eds.), Casebook of Clinical Hypnosis. Washington, D.C.: American Psychological Association. https://doi.org/10.1037/11090-011

Greer, H. S., y Engs, R. (1986). Use of progressive relaxation and hypnosis to increase tennis skill learning. Perceptual and Motor Skills, 63, 161-162. https://doi.org/10.2466/pms.1986.63.1.161 


\section{Hipnosis en Psicología del Deporte}

Gonsalkorale W. M., Houghton, L. A., y Whorwell, P. J. (2002). Hypnotherapy in irritable bowel syndrome: A large-scale audit of a clinical service with examination of factors influencing responsiveness. American Journal of Gastroenterology, 97, 954-61. https://doi.org/10.1111/j.1572-0241.2002.05615.x

Gonsalkorale, W. M., y Whorwell, P. J. (2005). Hypnotherapy in the treatment of irritable bowel syndrome. European Journal of Gastroenterology and Hepatology, 17, 15-20. https://doi.org/10.1097/00042737-200501000-00004

Gur, R. C. (1978). Measuring hypnotic susceptibility: a guest editorial. American Journal of Clinical Hypnosis, 21 , 64-67. https://doi.org/10.1080/00029157.1978.10403964

Hernández-Mendo, A., Delgado-Giralt, J., Fernández de Motta, M. M. y Carranque-Chaves, G. (2017). Eficacia de la hipnosis en psicología del deporte. Importancia de la comunicación y estudio de casos. Cuadernos de Psicología del Deporte, 17(3), 73-94. https://doi.org/10.6018/cpd.343401

Hilgard, E. R. (1977). Divided consciousness: Multiple controls in human thought and action. New York: John Wiley.

Howard, W. L., y Reardon, J. P. (1986). Changes in the Self Concept and Athletic Performance of Weight Lifters Through a Cognitive-Hypnotic Approach: An Empirical Study. American Journal of Clinical Hypnosis, $28(4), \quad 248-257$. https://doi.org/10.1080/00029157.1986.10402661

Jalene, S., y Wulf, G. (2014). Brief Hypnotic Intervention Increases Throwing Accuracy. International Journal of Sports Science y Coaching, 9(1), 199-206. https://doi.org/10.1260/17479541.9.1.199

Jackson, J. A., Gass, G. C., y Camp, El. M. (1979). The relationship between posthypnotic suggestion and endurance in physically trained subjects. International Journal of Clinical and Experimental Hypnosis, 27(3), 278-293.

https://doi.org/10.1080/00207147908407567

Kihlstrom, J. F. (1998). Hypnosis and the psychological Unconscious. Encyclopedia of Mental Health, 2, 467-477.

Ledesma, R., Macbeth, G. y Cortada de Kohan, N. (2008). Tamaño del efecto: Revisión teórica $\mathrm{y}$ aplicaciones con el sistema estadístico ViSta. Revista Latinoamericana de Psicología, 40(3), 425-439.

Levitt, E. E., y Brady, J. P. (1964). Muscular endurance under hypnosis and in the motivated waking state. The International Journal of Clinical and Experimental Hypnosis, 12(1), 21-27. https://doi.org/10.2466/pms.1975.40.1.131

Lindsay, P., Maynard, I., y Thomas, O. (2005). Effects of Hypnosis on Flow States and Cycling Performance. The Sport Psychologist, 19, 164-177. https://doi.org/10.1123/tsp.19.2.164

London, P., y Fuhrer, M. (1961). Hypnosis, motivation, and performance. Journal of Personality, 29(3), 321-333. https://doi.org/10.1111/j.14676494.1961.tb01665.x

London, P., Ogle, M. E., y Unikel, I. P. (1968). Effects of hypnosis and motivation on resistance to heat stress. Journal of Abnormal Psychology, 73(6), 532-541. https://doi.org/10.1037/h0026532

Lynn, S. J., Kirsh, I., Barabasz, A., Cardeña, E. y Patterson, D. (2000). Hypnosis as an empirically supported clinical intervention: The state of the evidence and a look to the future. International Journal of Clinical and Experimental Hypnosis, 48, 239-259.

https://doi.org/10.1080/00207140008410050

Lynn, S. J., Neufeld, V., Rhue, J. W., y Matorin, A. (1993). Hypnosis and smoking cessation: A cognitivebehavioral treatment. En J.W. Rhue, S.J. Lynn, y I. Kirsch (Eds.), Handbook of clinical hypnosis (pp. 555- 586). Washington, D.C.: American Psychological

Association.

https://doi.org/10.1037/10274-025

Lynn, S. J. y Shindler, K. (2002). The Role of Hypnotizability Assessment in Treatment. The American Journal of Clinical Hypnosis, 44, 185-197. https://doi.org/10.1080/00029157.2002.10403479

Mattle, S., Birrer, D., y Elfering, A. (2020). Feasibility of Hypnosis on Performance in Air Rifle Shooting Competition. International Journal of Clinical and Experimental Hypnosis, 68(4), 521-529. https://doi.org/10.1080/00207144.2020.1799655

Mattle, S., Birrer, D., y Elfering, A. (2020). Feasibility of Hypnosis on Performance in Air Rifle Shooting Competition. International Journal of Clinical and 


\section{Franquelo et al.}

Experimental Hypnosis, 68(4), 521-529. https://doi.org/10.1080/00207144.2020.1799655

Mendoza, M. E. (2000). La hipnosis como adjunto en el tratamiento del hábito de fumar: estudio de caso. Psicothema, 12, 330-338.

Molina-del-Peral, J. A., Mendoza, M. E., Capafons, A., y Llanes-Basulto, Y. (2016a). Hipnosis clínica: Evaluación de su eficacia y calidad de la investigación en psicología. Revista del Hospital Psiquiátrico de la Habana, 13(2). https://doi.org/10.18800/psico.201501.005

Molina del Peral, J. A., Mendoza, M. E., DíezGonzález, L., Llanes-Basulto, Y., y Capafons, A. (2016b). Hipnosis clínica: Evaluación de su eficacia y calidad de la investigación en medicina. Revista del Hospital Psiquiátrico de La Habana, 13(3). https://doi.org/10.1016/s0210-5691(06)74490-4

Montgomery, G. H., David, D., Winkel, G., Silverstein, J., y Bovbjerg, D. (2002). The effectiveness of adjunctive hypnosis with surgical patients: A meta-analysis. Anesthesia and Analgesia, 94, 1639-1645. https://doi.org/10.1213/00000539200206000-00052

Montgomery, G. H., DuHamel, K. N., y Reed, W. H. (2000). A meta-analysis of hypnotically induced analgesia: How effective is hypnosis? International Journal of Clinical and Experimental Hypnosis, 48, 138-153.https://doi.org/10.1080/00207140008410045

Montgomery, G. H., Schnur, J. B., y David, D. (2011). The Impact of Hypnotic Suggestibility In Clinical Care Settings. International Journal of Clinical and Experimental Hypnosis, 59(3), 294-309. https://doi.org/10.1080/00207144.2011.570656

Morgan, W. P., Peter, B. R., Drinkwater, B. L., y Horvath, S. M. (1973). Perceptual and metabolic responsivity to standard bicycle ergometry following various hypnotic suggestions. The International Journal of Clinical and Experimental Hpynosis, 21(2), 86-101. https://doi.org/10.1080/00207147308409309

Morton, P. A. (2003) The Hypnotic Belay in Alpine Mountaineering: The Use of Self-Hypnosis for the Resolution of Sports Injuries and for Performance Enhancement. American Journal of Clinical Hypnosis, 46(1), 45-51. https://doi.org/10.1080/00029157.2003.10403564
Newmark, T. S., y Bogacki, D. F. (2005). The Use of Relaxation, Hypnosis, and Imaginery in Sport Psychiatry. Clinics in Sports Medicine, 24(4), 973977. https://doi.org/10.1016/j.csm.2005.06.003

Nelson, J. (1980). Investigation of Effects of Hypnosis, Relaxation, and Mental Rehearsal on Performance Scores of Golfers and Runners.

Orne, M. T. (1959). The Nature of Hypnosis: Artifact and Essence. Journal of Abnormal and Social Psychology, 58, 277-299. https://doi.org/10.1037/h0046128

Palsson, O. S. (2006). Standardized hypnosis treatment for irritable bowel syndrome: The North Carolina protocol. International Journal of Clinical and Experimental Hypnosis, 54, 51-64. https://doi.org/10.1080/00207140500322933

Pates, J. (2013). The Effects of Hypnosis on an Elite Senior European Tour Golfer: A Single-Subject Design. International Journal of Clinical and Experimental Hypnosis, 61(2), 193-204. https://doi.org/10.1080/00207144.2013.753831

Pates, J. (2014). The Effects of Hypnosis on an Elite Senior European Tour Golfer. Journal of Excellence, 16, 74-83.

Pates, J. (2019). Hypnosis in the Clutch. Biomedical Journal of Scientific and Technical Research, 23(2), 17205-17211.

https://doi.org/10.26717/BJSTR.2019.23.003863

Pates, J., Cummings, A., y Maynard, I. (2002). The Effects of Hypnosis on Flow States and Three-Point Shooting Performance in Basketball Players. The Sport Psychologist, 16, 34-47. https://doi.org/10.1123/tsp.16.1.34

Pates, J., y Cowen, A. (2013). The Effect of a Hypnosis Intervention on Performance and Flow State of an Elite Golfer: A Single Subject Design. International Journal of Golf Science, 2, 43-53. https://doi.org/10.1123/ijgs.2.1.43

Pates, J. y Maynard, I. (2000). Effects of hypnosis on flow states and golf performance. Perceptual and Motor Skills, 91, 1057-1075. https://doi.org/10.2466/pms.2000.91.3f.1057

Pates, J., Maynard, I., y Westbury, T. (2001). An Investigation into the Effects of Hypnosis on Basketball Performance. Journal of Applied Sport 


\section{Hipnosis en Psicología del Deporte}

Psychology, 13(1), 84-102. https://doi.org/10.1080/10413200109339005

Pates, J., Oliver, R., y Maynard, I. (2001). The Effects of Hypnosis on Flow States and Golf-Putting Performance. Journal of Applied Sport Psychology, 13 , 341-354.

https://doi.org/10.1080/104132001753226238

Pates, J. y Palmi, J. (2002). The Effects of Hypnosis on Flow States and Performance. Journal of Excellence, 6, 48-62.

Pinnell, C. M., y Covino, N. A. (2000) Empirical findings on the use of hypnosis in medicine: A critical review. International Journal of Clinical and Experimental Hypnosis, 48, 170-194. https://doi.org/10.1080/00207140008410047

Rader, C. M. (1972). Influence of motivational instructions on hypnotic and nonhypnotic reaction time performance. The American Journal of Clinical Hypnosis, $\quad 15, \quad 98-\quad 101$. https://doi.org/10.1080/00029157.1972.10402226

Robazza, C, y Bortoli, L. (1995). A case study of improved performance in archery using hypnosis. Perceptual and Motor Skills, 81, 1364-1366. https://doi.org/10.2466/pms.1995.81.3f.1364

Rosenhan, D., y London, P. (1963). Hypnosis: expectation, susceptibility and performance. Journal of Abnormal and Social Psychology, 66(1), 77-81. https://doi.org/10.1037/h0043303

Roush, E. S. (1951). Strength and endurance in the waking and hypnotic states. Journal of Applied Physiology, 3, 404-410. https://doi.org/10.1152/jappl.1951.3.7.404

Schoenberger, N. E., Kirsch, I., Gearan, P., Montgomery, G., y Pastyrnak, S. L., (1997). Hypnotic enhancement of a cognitive behavioral treatment for public speaking anxiety. Behavior Therapy, 28(1), 127-140. $\quad$ https://doi.org/10.1016/S00057894(97)80038-X

Schreiber, E. H. (1991). Using hypnosis to improve performance of college basketball players'. Perceptual and Motor Skills, 72, 536-538.
$\underline{\text { https://doi.org/10.2466/pms.1991.72.2.536 }}$

Simrén, M. (2006). Hypnosis for irritable bowel syndrome: The quest for the mechanism of action. International Journal of Clinical and Experimental Hypnosis, $\quad 54, \quad 65-84$. https://doi.org/10.1080/00207140500327239

Spiegel, H. y Spiegel, D. (1987) Trance and Treatment: Clinical Uses of Hypnosis. 2nd Edition, Washington, DC: American Psychiatric Press. https://doi.org/10.1080/00207140591001794

Whitehead, W. E. (2006). Hypnosis for irritable bowel syndrome: The empirical evidence of therapeutic effects. International Journal of Clinical and Experimental Hypnosis, 54, 7-20. https://doi.org/10.1080/00207140500328708

Whorwell, P. J. (2006). Effective management of irritable bowel syndrome-the Manchester Model. International Journal of Clinical and Experimental Hypnosis, $\quad 54, \quad 21-26$. https://doi.org/10.1080/00207140500323006

Winter, G. (1986). The use of hypnosis in an applied sport psychology setting. Behaviour Change, 3(2), 120-126.

https://doi.org/10.1017/s081348390000927x

Young, P. C. (1925). An Experimental Study of Mental and Physical Functions in the Normal and Hypnotic States. The American Journal of Psychology, 36(2), 214-232. https://doi.org/10.2307/1413859

Zurita, F., Fernández, R., Pérez, A. J., y Castro, M. (2014). Hipnosis en la mejora de fuerza y resistencia. Publicaciones, 44, 89-112. 